\title{
EXPLORANDO POTENCIALES VÍNCULOS ENTRE RESILENCIA Y JUSTICIA AMBIENTAL: EL CASO DE CHAITÉN, CHILE
}

\author{
VICENTE SANDOVAL, , c CAMILO BOANO ${ }^{\mathrm{a}}$, \\ CLAUDIA GONZÁLEZ-MUZZIO ${ }^{b}$ \& CRISTIAN ALBORNOZc
}

\begin{abstract}
RESUMEN
En mayo de 2008, la ciudad de Chaitén fue evacuada debido al riesgo de una erupción volcánica. Días más tarde, graves inundaciones como consecuencia de la erupción produjeron su casi total desaparición. En los meses siguientes, el Estado desarrolló diversos planes para la reubicación de Chaitén, así como estrategias para promover la resiliencia y recuperar a la población. Planes y estrategias que, de aquel tiempo a esta parte, no obtuvieron los resultados esperados, perjudicado implícitamente a la población y su capacidad de resistir, adaptarse a, y recuperarse de futuros desastres. A siete años de la erupción, este trabajo explora la asimetría espacial, socio-económica y ambiental de la ciudad de Chaitén, explicada en parte a la erupción volcánica, pero también (y más importante aún) a decisiones gubernamentales 'erráticas' y a los efectos 'inesperados' de las políticas diseñadas para recuperar a la población. Algunas de las razones para explicar la situación actual de Chaitén, son la 'improvisación' con la que se abordó la evacuación y el programa de recuperación, así como la 'indecisión' del Estado sobre la reubicación de la localidad. De esta manera, Chaitén se ha constituido en una ciudad desigual en términos de justicia ambiental, en donde además de otras asimetrías, los efectos de las política públicas se distribuyen de manera inequitativa. En este sentido, este trabajo indaga en la manera en como los procesos diseñados para incrementar la resiliencia de una población pueden, por el contrario, producir un impacto negativo en la justicia ambiental, y por lo tanto, producir nuevas situaciones de vulnerabilidad y riesgo.
\end{abstract}

PALABRAS CLAVE: Chaitén, resiliencia, justicia ambiental, distribución desigual, desastre.

\section{A POTENTIAL RELATIONSHIP BETWEEN RESILIENCE AND ENVIRONMENTAL JUSTICE:THE CASE OF CHAITÉN, CHILE}

\footnotetext{
ABSTRACT

In May 2008, the city of Chaitén was completely evacuated due to the probability of a volcanic eruption. Few days later, the eruption caused severe flooding that almost destroyed the entire city. In

a The Bartlett Development Planning Unit (DPU), University College London (UCL), 34 Tevistock Square, WC1H 9EZ Londres, Reino Unido. vicente.sandoval.11@ucl.ac.uk, c.boano@ucl.ac.uk

b_Ambito Consultores Ltda., Santiago, Chile. cgonzalez@ambito.cl

c Centro de Investigación Vulnerabilidades y Desastres Socio-naturales (CIVDES), Universidad de Chile, Avenida Capitán Ignacio Carrera Pinto 1045, Ñuñoa, Santiago, Chile. albornoz@stud.uni-heidelberg.de
} 
the following months, the state developed plans for the relocation of Chaiten, and strategies to promote resilience and the recovery of population. However, plans and strategies resulted in unexpected results impacting people's ability to resist, adapt to, and recover from future disasters. This paper explores a potential asymmetric relationship in the distribution of environmental risks and resilience within Chaitén. Such relationship can be partially explained by the volcanic eruption, but more importantly, by unforeseen effects of decision making and policies implemented for the recovery of the affected population. Effects of the implemented benefit schemes and decision making related to the inhabitability/relocation of Chaitén can offer some explanation about the current situation of Chaitén; a highly segregated and environmentally unjust city where public policies' outputs are unevenly distributed. In this sense, this paper examines how plans and policies developed towards increasing resilience among population may, inversely, impact negatively on environmental justice, that is, on the distribution of environmental risks, exposure and disaster impacts.

KEY WORDS: Chaitén, resilience, environmental justice, uneven distribution, disaster.

\section{INTRODUCCIÓN}

Este artículo ${ }^{1}$ emplea el caso de Chaitén para discutir potenciales vínculos entre los procesos de resiliencia y justicia ambiental. La justicia ambiental es entendida aquí como la distribución equitativa de los riesgos, pero también como el acceso equitativo a bienes y servicios que dependen de los recursos naturales -ej. agua potable, energía-, al igual que a un sistema urbano y/o rural adecuado para el desarrollo humano, incluyendo un apropiado entorno construido y la disponibilidad de áreas verdes (Walker \& Bulkeley, 2006). Por otra parte, la resiliencia se entiende como la habilidad de un sistema social de responder y recuperarse de los desastres, que incluye aquellas condiciones inherentes que permiten al sistema absorber impactos y enfrentar los efectos negativos posteriores a un evento destructivo, así como también las procesos adaptativos que facilitan la habilidad del sistema social de reorganizarse, cambiar y aprender en respuesta a una amenaza (Cutter et al. 2008).

De esta manera, el trabajo se orienta a entender mejor el caso de Chaitén, una comunidad fuertemente afectada por una erupción volcánica el año 2008, a través de la exploración de procesos sociales y políticos como la toma de decisiones por parte de las autoridades a distintas escalas geográficas (local, regional y nacional) y sus efectos concretos y localizados en la comunidad de Chaitén; cómo reaccionaron las autoridades y la población frente a los efectos de la erupción

1 Este documento recoge el trabajo colaborativo de proyectos de consultoría, estudios académicos $y$ observaciones realizados por los autores a partir del volcánica, cómo se organizaron las instituciones para intentar recuperar a la población afectada, cómo se adaptaron y aprendieron del evento, entre otras interrogantes son parte de este trabajo.

Hacia el final de este artículo, la pregunta sobre la actual situación de Chaitén, la de una ciudad dividida, es abordada críticamente y analizada bajo el lente de la coproducción entre los procesos de resiliencia y justicia ambiental.

En términos de relación entre resiliencia y justicia ambiental, se argumenta que las acciones posteriores al desastre adoptadas por las autoridades y la comunidad, relacionadas con procesos que buscaban incrementar la capacidad de la población para resistir y adaptarse a futuros eventos, pueden haber afectado negativamente la justicia ambiental. De este modo, este trabajo explora una posible relación inversa entre resiliencia y justicia ambiental (Schilderman \& Lyons, 2010). Asimismo, se sostiene que las políticas adoptadas han llevado en gran medida a la desigual distribución de los riesgos y el acceso limitado a los servicios básicos de la población de Chaitén. Finalmente, este trabajo ilustra cómo estas desigualdades -las cuales no existían antes de la erupción del volcán- actualmente se manifiestan de manera abrupta y evidente. Se postula que, en consecuencia, los chaiteninos son más vulnerables a los desastres que antes de 2008.

La metodología de trabajo fue principalmente cualitativa e incluía métodos de investigación

2011, así como entrevistas a autoridades y líderes locales, $y$ diversos grupos focales realizados entre marzo y julio de 2013. 
social tanto en los tres viajes de campo durante el 2013 (Santiago, Puerto Montt y Chaitén) como en la revisión de material documental, incluyendo entrevistas en profundidad, observación participativa y no-participativa, revisión y análisis de informes y documento de gobierno $\mathrm{y}$ otras instituciones estatales, revisión de material de prensa y entrevistas a autoridades en medios de comunicación. Todo este trabajo se realizó entre Enero de 2013 y Diciembre de 2014, y contempla observaciones y experiencias recogidas a través de trabajos de consultaría y estudios académicos realizados por los autores a partir del 2011.

\section{CONTEXTO: EL DESASTRE DE CHAITÉN.}

En mayo de 2008, el volcán Chaitén situado en la Región de Los Lagos entró en erupción, causando la evacuación de gran parte de la población de la comuna, alrededor de 8.000 habitantes (De la Barrera et al. 2011) y algunas otras localidades de la provincia de Palena. La población fue evacuada principalmente a las ciudades de los alrededores, como Puerto Montt y Castro, todas ellas situadas a más de $100 \mathrm{~km}$ por mar. Varios días después de la evacuación, lahares ${ }^{2}$ y fuertes lluvias causaron graves inundaciones que dejaron a Chaitén inhabitable y prácticamente devastada (Lara, 2009). La ciudad quedó dividida en el sector norte y sur por el nuevo curso del río Blanco (Fig 1). Las pérdidas económicas se estimaron en US\$12 millones, únicamente en edificios públicos asegurados (Lara, 2009). Mientras que aún no hay claridad sobre el costo total de la catástrofe, las autoridades calculan que entre US\$ 60 y 80 millones se gastaron durante el primer año y que se emplearon más US\$ 3 millones para reconstruir la infraestructura pública dañada (Presidencia de la República de Chile, 2009; Galilea, 2009).

El desastre no sólo afectó a Chaitén, capital de la Provincia de Palena, sino también a toda la provincia, la cual contaba con 18.971 habitantes para entonces (INE 2002). El Gobierno Provincial tuvo que ser trasladado temporalmente a Futaleufú. Del mismo modo, la Municipalidad de Chaitén funcionó 'virtualmente' desde Puerto Montt hasta el año 2011, cuando el gobierno provincial y el municipio se

2 Los lahares son una mezcla de rocas fragmentadas preexistentes en las laderas de los volcanes, materiales efusivos frescos, con una gran cantidad de agua, que reinstalaron en Chaitén.

Tres informes encargados por el Gobierno de Chile (Moreno \& Lara, 2008; Moreno et al. 2008; SERNAGEOMIN, 2009) consideraron Chaitén como altamente propenso a nuevas erupciones y actividad sísmica. A partir de dichos estudios, en febrero de 2009, el Ministerio del Interior Edmundo Pérez Yoma anunció por los medios de comunicación que desafortunadamente, Chaitén ha muerto (La Nación, 2009). Este anuncio implicó que ni la reconstrucción de Chaitén, ni más inversión de ningún tipo en la ubicación actual se llevarían a cabo. Por consiguiente, se consideraron planes alternativos basados en el desplazamiento de Chaitén a zonas más seguras. A finales de 2009, Santa Bárbara fue elegida como ubicación de la Nueva Chaitén. El Ministerio de Vivienda y Urbanismo (MINVU), basado en un estudio de la Pontificia Universidad Católica de Chile et al. (2009), desarrolló para ello un Plan Maestro el 2010, de forma que la nueva ciudad se construiría en tres etapas, la primera para ser completada a finales del 2012 (MINVU, 2010). Sin embargo, en febrero de ese mismo año, un terremoto de gran intensidad sacudió la zona centro-sur de Chile, matando a unas 500 personas y afectando a más de dos millones de habitantes, causando pérdidas económicas por un total de US\$ 30 mil millones (EM-DAT, 2015). A partir de este evento, los planes para la Nueva Chaitén dejaron de ser una prioridad (Ramírez, 2010).

A pesar del cambio político de un gobierno de izquierda a uno de derecha en marzo de 2010, el entonces Presidente electo Sebastián Piñera afirmó ese mismo año que Chaitén debía ser reubicada, confirmando así la decisión de la administración anterior. Contradictoriamente, a fines del 2010 el sector norte de Chaitén fue declarado 'habitable' nuevamente. El Gobierno declaró que este drástico cambio se basó en estudios técnicos que sugerían tomar diversas medidas de mitigación para reducir la exposición de la población (Municipalidad de Chaitén, 2011), pero que también fue en respuesta a la demanda de más de 500 chaiteninos que habían regresado de manera informal a la ciudad, resistiendo cualquier intento de desalojo y reubicación (Rojas, 2013). De acuerdo con la población local:

provienen de la fusión instantánea de cubiertas de hielo y nieve por efecto de un evento eruptivo o por condiciones de intensas precipitaciones (González-Ferrán, 1995, p. 60). 


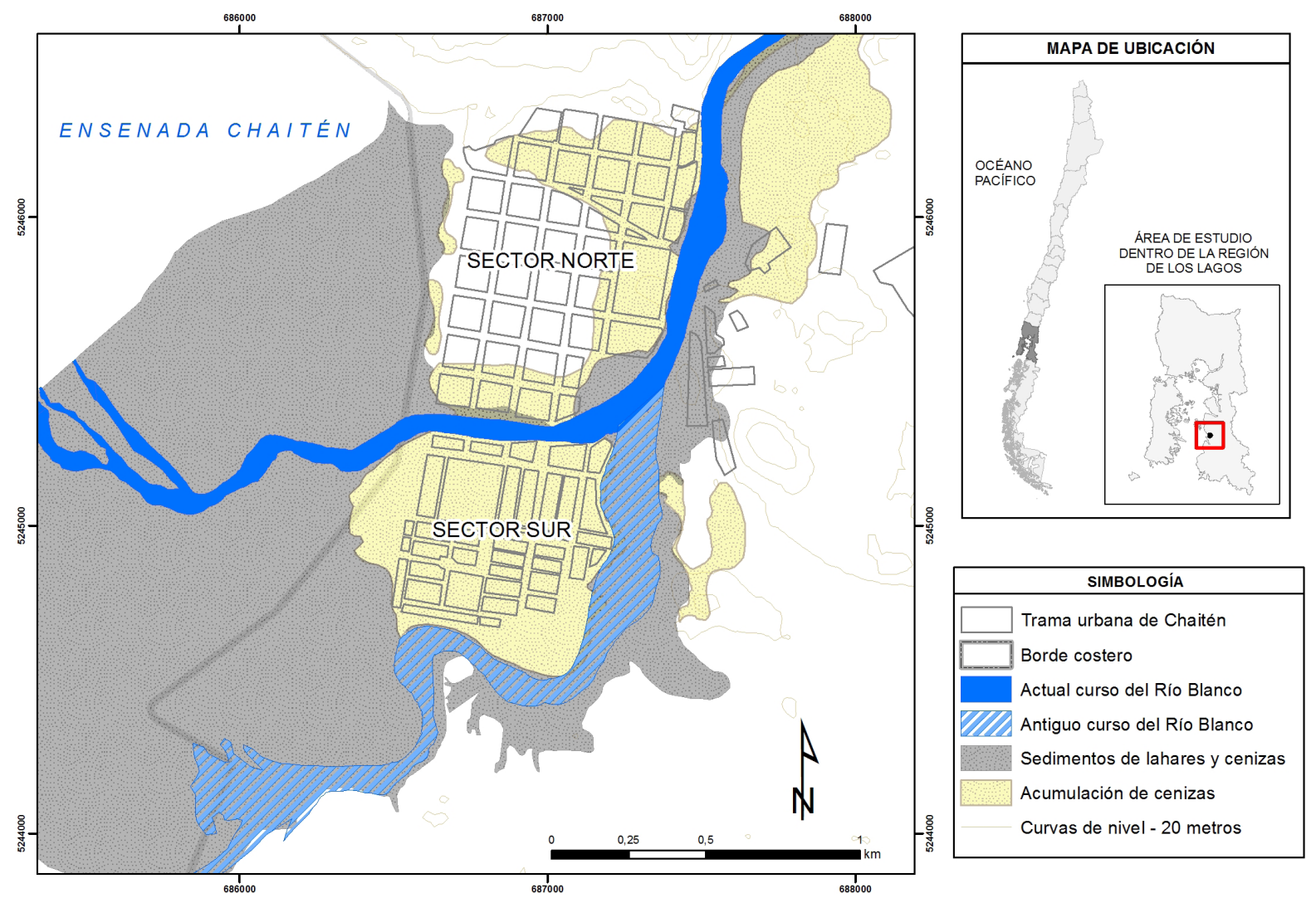

Fig. 1. Mapa de Chaitén y el río Blanco, antes y después de 2008.

Fuente: Elaboración propia basado en INFRACON S.A. (2012)

Fue una tremenda lucha contra las intenciones del gobierno para trasladarnos definitivamente [...]. Estuvimos firmes y fuertes porque Chaitén ha sido siempre nuestra tierra; no queríamos vivir en otro sitio. (Líder local anónimo, 2013, com. pers.)

Al declarar 'habitable' nuevamente Chaitén, pero sólo su sector norte, la ciudad quedó dividida no sólo por el nuevo cauce del río Blanco, sino también, y más importante, por una regulación de uso de suelo determinada por el gobierno central. Según las autoridades locales, hacia finales del 2013 más de 160 familias habitaban de manera informal el sector sur. De acuerdo a las observaciones y entrevistas realizadas en el lugar, dichas familias viven en una situación precaria, enfrentando dificultades para acceder a los

3 La última erupción del volcán Chaitén anterior al 2008 data alrededor de 9,370 BP (Lara, 2009; Naranjo \& servicios básicos como agua potable, electricidad o alcantarillado, así como a la salud, la asistencia social y la educación. Aunque no hay registros oficiales sobre la población total de Chaitén, las autoridades municipales afirmaban que en 2013 alrededor de 2.500 personas habitaban la ciudad, incluyendo aquellos del sector sur.

\section{PROMOVIENDO LA RESILIENCIA DESDE EL ESTADO: INSTALACIÓN DE UNA DESIGUAL DISTRIBUCIÓN DE RIESGOS Y SERVICIOS URBANOS}

Aunque Chaitén se encuentra cerca de varios volcanes activos (INFRACON S.A., 2012), los habitantes y autoridades no eran conscientes de los riesgos volcánicos ${ }^{3}$, por lo que cuando se produjo la erupción, no había un plan de evacuación. Sin 
embargo, en 24 horas 4.101 personas fueron evacuadas por mar -tanto por la Armada de Chile (marina) como por barcos privados- $y$, en pocos días, más de 8.000 personas abandonaron la zona (Presidencia de la República de Chile, 2009).

El 2 de mayo de 2008, el área fue declarada como Zona de Catástrofe (GORE Los Lagos, 2008). Esta medida era aplicable por un año, extensible por un período igual, y estuvo vigente en Chaitén hasta finales del 2010. Durante dicho periodo, se asignaron varios recursos para apoyar a la población desplazada, así como la creación de una autoridad especial, llamada "Delegada Presidencial", encargada de velar por el buen desarrollo del proceso.

Las principales medidas incluidas eran bonos mensuales de hasta US\$1.000 por familia, los que se entregaron a más de 3.200 familias durante el primer año y a alrededor de 1.800 familias el segundo año; bonos de compensación para los agricultores; subvenciones para la educación de los niños y jóvenes; subsidios de vivienda por alrededor de US\$20.000 dólares para 2.235 familias; subsidios a la iniciativa empresarial y recursos destinados al apoyo psicológico; entre otros (GORE Los Lagos, 2009, 2010; Presidencia de la República de Chile, 2009).

Asimismo, se implementó la Ley especial (Ley $\mathrm{N}^{\circ}$ 20.385) llamada "Ley Chaitén" que permitió al Estado comprar propiedades en Chaitén pagando por ellas su precio de mercado antes de la erupción. Una vez aprobada, el Estado compró 889 propiedades por un total de US\$ 30 millones (El Mercurio, 2013). Más del 75\% del parque de viviendas en Chaitén es ahora propiedad pública. Cerca de 70 viviendas fueron alquiladas por algunos habitantes del sector norte. Otros, que no vendieron sus propiedades a través de esta ley, cuando regresaron a Chaitén lo hicieron ocupando sus propias casas. Además, se asignaron 49 propiedades a oficinas públicas. Desde el 2014 , el Estado oferta una centena de propiedades en venta, principalmente a sus anteriores dueños (Ibid.). No obstante en el sector sur, sigue siendo ilegal habitar. Declarado como área no-apta para vivir debido a la amenaza de nuevas inundaciones (o la falta de infraestructura para mitigar tal amenaza), en el sector sur no hay permisos para arrendar y re-comprar las propiedades, incluso por sus antiguos dueños, por lo que los inmuebles son ocupados ilegalmente pese a que un número importante de personas habitan, desarrollan sus medios de subsistencia, y llevan una vida con relativa normalidad alli (Rojas, 2013).

Hacia principios del 2010, el Gobierno impulsó el desarrollo de un plan para reconstruir una nueva ciudad para los chaiteninos. Este fue el "Plan Maestro para la Nueva Chaitén" (MINVU, 2010), el cual tenía un costo de implementación evaluado en US\$300 millones (Silva, 2010). Aunque finalmente se construyeron algunas infraestructuras públicas en Santa Bárbara, por ejemplo para la Dirección General de Aeronáutica Civil, Carabineros y otras oficinas públicas, no se llevó a cabo ninguna otra acción de relevancia. En mayo de 2010, las autoridades regionales anunciaron que descartarían la Nueva Chaitén debido a su costo y el rechazo de la población local (Municipalidad de Chaitén, 2011). Como prueba de falta de coordinación, días después de ese anuncio, el Presidente de la República Sebastian Piñera corregía la declaración del entonces Intendente de la Región de Los Lagos Juan Sebastián Montes, e insistía en que la Nueva Chaitén se construiría de todos modos (El Mercurio, 2010). Irónicamente, en diciembre de 2010 el sector norte de Chaitén fue definitivamente declarado "habitable" (Municipalidad de Chaitén, 2011).

Con el fin de decidir si Chaitén se podría habitar nuevamente, el Gobierno encargó otro estudio que concluyó que la población local podría vivir cerca de las cenizas volcánicas siempre y cuando se adoptaran medidas de mitigación, restauración y vigilancia ambiental que permitieran reducir el impacto negativo de las cenizas, así como de futuras inundaciones (CIMM T S Consultores, 2010). Además, la actividad volcánica fue disminuyendo y algunas áreas no estaban en riesgo de nuevas inundaciones o lahares (Intendencia Región de Los Lagos, 2010) (Fig. 2).

De este modo, al declarar habitable Chaitén norte, el proyecto de reubicación -de iniciativa pública- fue abortado $\mathrm{y}$, por consiguiente, la responsabilidad de tomar la decisión de reasentarse en un lugar anteriormente declarado como de alto riesgo fue traspasada a la población local. Con estas palabras el Gobierno de Sebastián Piñera valoraba el "espíritu emprendedor" de los chaiteninos y su 


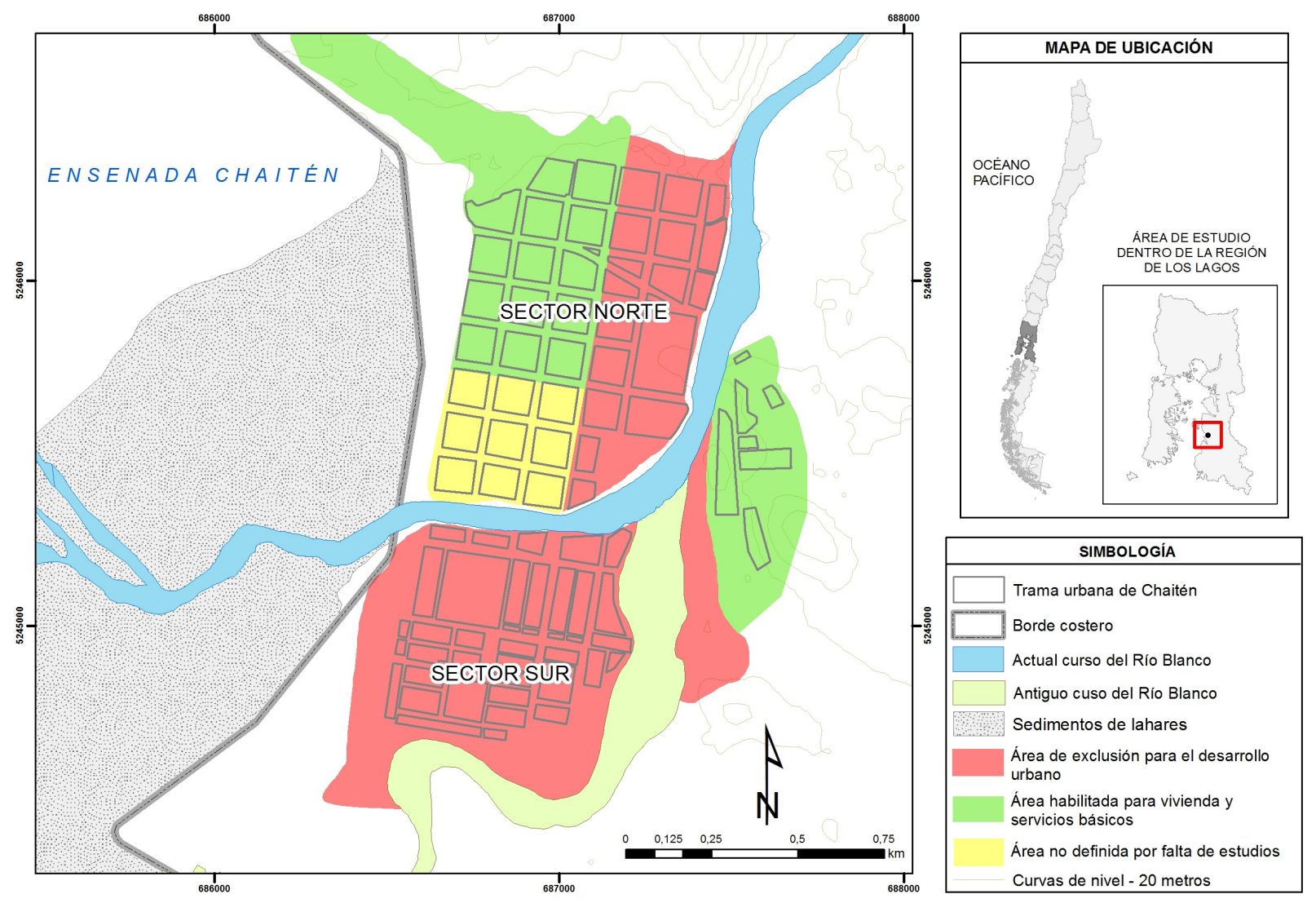

Fig. 2. Análisis de las áreas expuestas y su designación.

Fuente: Elaboración propia basado en CIMM TS Consultores (2010) y Municipalidad de Chaitén (2011).

insistencia de vivir en Chaitén:

El Gobierno tiene la convicción de que las ciudades han de nacer y crecer por procesos evolutivos normales, con el apoyo de la población local y no bajo la voluntad y el apoyo mayoritario del Estado [...]. Santa Bárbara sería hoy una empresa financiada y dirigida exclusivamente por el Estado, sin evidencia de apoyo de un grupo importante de la población local para acompañar dicho emprendimiento de reubicación. Por otra parte, los actuales habitantes de Chaitén, en una actitud firme y valiente, han asumido en base a su libertad y responsabilidad, la decisión de habitar sus tierras y quedarse ahí, lo cual este Gobierno valora y reconoce como el espíritu que debe animar las decisiones de asentamiento territorial en la región. (Intendencia Región de Los Lagos, 2010).
Al examinar algunas de las respuestas del Estado, se puede argumentar que aunque apuntaban a mejorar las condiciones de vida de la población, su recuperación y resiliencia, así como también disminuir los niveles de vulnerabilidad, las acciones (o falta de ellas) del Estado han sido contraproducentes. Éstas respuestas han tendido a exacerbar la vulnerabilidad entre la población de Chaitén, propiciando una distribución desigual del riesgo y afectando negativamente la resiliencia de la población.

\section{LAS RESPUESTAS DE LA COMUNIDAD, REACCIONANDO AL ESTADO}

La escasa claridad con respecto a las causas de la actividad sísmica en la zona con anterioridad a la erupción, así como el desconocimiento sobre los riesgos y medidas de emergencia necesarias para proteger a la población, pueden servir de 
ejemplo para entender la pérdida de confianza en las autoridades por parte de los chaiteninos. Tal y como declaró un líder local, la reunión entre representantes de la población de Chaitén y el gobierno regional pocos días antes de la erupción fue desorganizada:

La situación sobre los temblores era tan poco clara y confusa que la conversación [entre la comunidad y el Gobierno] derivó hacia a los efectos de la sequía en la región. (Líder local anónimo, 2013, com. pers).

Finalmente, la mayoría de la población aceptó la evacuación casi sin resistencia inmediatamente cuando las autoridades lo decidieron. Por tal razón, la evacuación de la ciudad fue considerada por las autoridades y la población como una medida preventiva adecuada al ponderar el riesgo de erupción. Sin embargo, los evacuados fueron guiados sin un orden predefinido y de manera dispersa a distintas ciudades de la región. En algunos casos, las familias se separaron porque las mujeres y los niños fueron evacuados primero. Por otra parte, la organización social existente fue desarticulada y no habían interlocutores capaces de representar a la población dispersa o de establecer comunicación con las autoridades regionales y locales (Mardones et al. 2011).

Con el tiempo, y para hacer frente a la incertidumbre, los chaiteninos generaron una organización comunitaria informal denominada "Hijos y amigos de Chaitén", con el fin de comunicar a las autoridades sus necesidades, así como su intención de regresar a la ciudad. Debido al bajo impacto mediático de las demandas de los chaiteninos, algunos de ellos decidieron retornar y ocupar Chaitén hacia finales de 2008, a pesar de la prohibición existente. La prensa los llamó "Los Rebeldes" (Rojas, 2013). Vivir en una ciudad abandonada como Chaitén significaba que tenían que arreglárselas para cubrir sus necesidades básicas. Obtuvieron agua potable a partir de un pequeño arroyo al que conectaron tuberías, mientras que la energía la obtenían a partir de un generador diésel. Los pobladores se organizaron para limpiar las calles y reparar sus casas. En estas condiciones, tuvieron que redefinir sus medios de vida y modificar sus estrategias de subsistencia para enfrentar la escasez de bienes y productos en la ciudad, sin comercio o puestos de trabajo disponibles.

A principios del 2009, los hijos e hijas de Los Rebeldes llegaron a la ciudad para acompañar a sus padres. Sin embargo, las autoridades regionales, quienes se negaban a dialogar con los nuevos colonos, intentaron convencerlos de abandonar Chaitén ordenando a la Armada (marina) sacar a los niños fuera de la ciudad nuevamente, argumentando que los menores eran ahora responsabilidad del Estado, dado que sus padres eran capaces de exponerlos 'irresponsablemente' a la amenaza del volcán. Según otro líder local ellos [las autoridades] creían que nosotros íbamos a seguir a nuestros hijos, y salir de Chaitén, pero no lo hicimos, tuvimos que enviarlos solos [...] lloramos por semanas, lejos de ellos [sus hijos] (2013, com. pers.). Los Rebeldes constituían lo que Quarantelli (2004) llama un grupo emergente, generado para reaccionar y responder a las consecuencias de un desastre. Como tal, se convirtieron en portavoces de la población dispersa de Chaitén. La comunicación entre chaiteninos exiliados, como se referían a quienes aún vivían fuera de Chaitén, fue posible gracias a un programa de radio llamado "Aquí Chaitén" emitido desde la ciudad por un vecino.

A pesar de las dificultades, estos colonos (como se auto-denominaban) se adaptaron a la nueva situación, mientras que al mismo tiempo alentaban a otros chaiteninos a regresar a la ciudad. Los Rebeldes percibían que los exiliados enfrentaban problemas psicológicos originados por los mismos especialistas designados por el gobierno central para apoyarlos en el proceso de reubicación. Según Los Rebeldes, a los psicólogos se les instruyó para convencer a la gente que Chaitén estaba muerto y que nunca podría habitar allí de nuevo (Líder local anónimo, 2013, com. pers.). Tanto Los Rebeldes como otros chaiteninos se refieren esta situación como un lavado de cerebro, promovido por las autoridades en un esfuerzo por eludir o hacer frente a las demandas de la población (Líder local anónimo, 2013, com. pers.). Lamentablemente no existen registros de tales intenciones, no obstante, la certeza y reiterado discurso sobre este asunto da cuenta de una clara desconfianza sobre las intenciones del gobierno y 


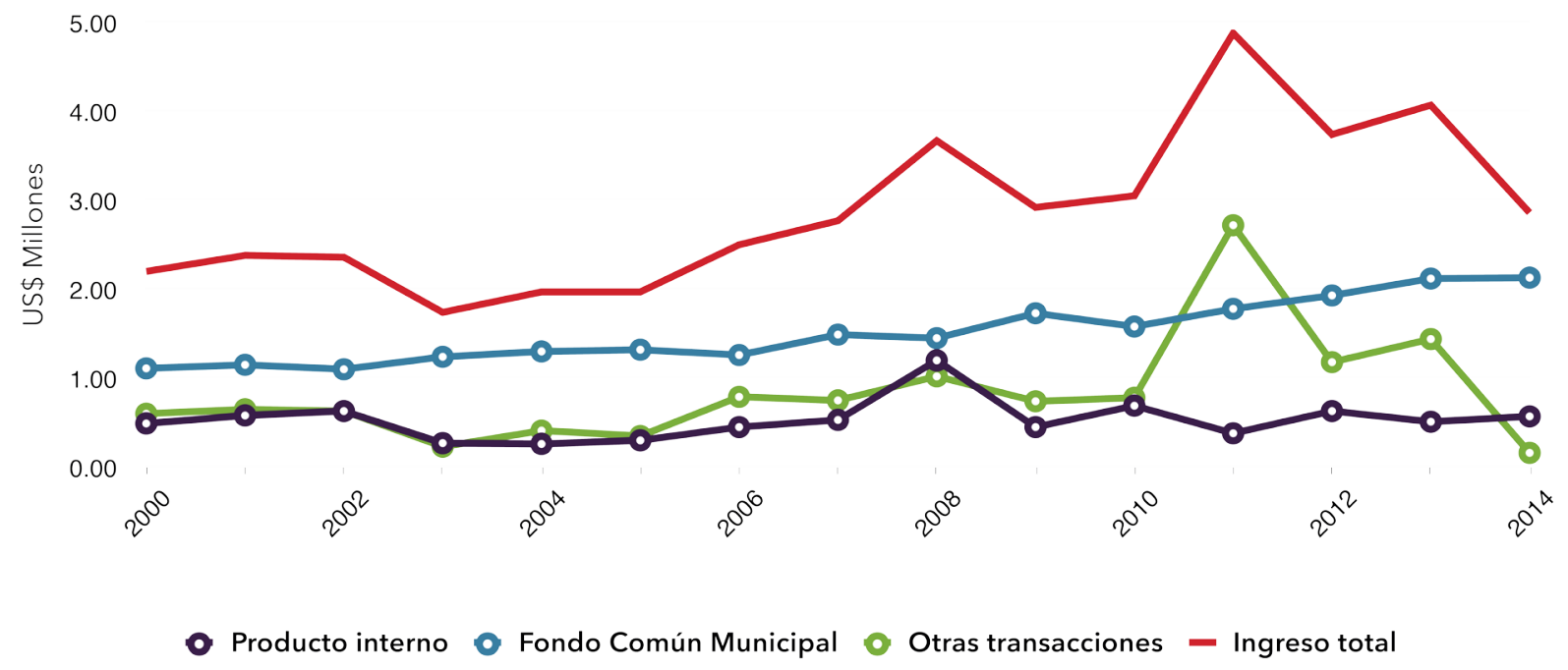

Fig. 3. Evolución del presupuesto comunal para Chaitén desde 2000 a 2014

Fuente: Elaboración propia basado en el Sistema Nacional de Información Municipal SINIM (2014)

de sus autoridades, un tema que se hace recurrente en casi todas las entrevistas y observaciones realizadas en Chaitén.

En 2010, cuando la decisión de reubicar la ciudad estaba todavía en curso, las autoridades argumentaron que era esencial establecer un diálogo con la comunidad para que el proyecto de la Nueva Chaitén se hiciera realidad (Cifuentes, 2009). Sin embargo, la comunidad consideró que la iniciativa llegó demasiado tarde y afirmaron que la Nueva Chaitén estaba sólo en la imaginación de los planificadores y las autoridades públicas. Las personas viviendo en Chaitén también argumentaron que no fueron consideradas en las reuniones de participación celebradas en relación al proyecto de reubicación. Por otro lado, el MINVU (2010) afirmaba que 460 personas desplazadas que vivían en 14 localidades participaron en el proceso de planificación. Chaitén no estaba en la lista de localidades consultadas pese a que entonces vivían alli más de 500 personas.

A finales de 2010, los servicios básicos fueron restaurados en el sector norte de Chaitén, el gobierno local y provincial retornaron a la ciudad y un nuevo plan de inversión fue anunciado para mejorar la infraestructura pública (Municipalidad de Chaitén, 2011). Aparentemente este plan no llegó a concretarse, dado que posterior al 2010 no existen documentos oficiales del gobierno que lo mencionen. No obstante, sí existió inversión, la cual se puede resumir en una suma de diferentes recursos y proyectos adjudicados entre finales de 2010 y 2012 orientados a mitigar el riesgo en Chaitén a través de proyectos de infraestructura, y se pueden observar en la evolución del presupuesto comunal desde el año del desastre (2008) hasta el 2014 (Fig. 3).

El primer colono del sector sur regresó en 2010 a su antigua casa, ya que decidió no venderla través de la llamada "Ley Chaitén". Para mediados de 2013, cerca de 160 familias ocupaban viviendas en el sector sur. Esta población argumentaba su asentamiento en el sector debido a la ausencia de soluciones habitacionales disponibles en el sector norte. Durante el 2008 y 2013, la población ha invertido en la reparación de casas, a pesar de la prohibición de habitar el sector y la carencia total de servicios públicos. Con todo, los residentes del sector sur se han organizado para conseguir agua y electricidad, prorrateando el costo (alrededor de US\$60 al mes por familia). Sin embargo, no existe una organización para satisfacer otras necesidades, como el transporte, la salud y la educación, asî como la búsqueda de medios de vida más seguros. Además, la comunidad del sector sur sigue viviendo en una especie de medio ambiente "de posguerra", rodeada de escombros producidos por la erupción el 2008, la reciente política de demolición de casas impartida por el gobierno central y regional, y la acumulación de desechos (Fig. 4). Muchas 
personas del sector sur viajan a pie diariamente el centro de la ciudad donde las cosas están volviendo a la normalidad, llegando a recorrer más de 5 kilómetros en cada viaje, mientras que aún ambos sectores demandan por un puente que conecte nuevamente el norte y el sur de la ciudad.

En ambos sectores de Chaitén, las organizaciones sociales han surgido principalmente para asegurar el acceso a los servicios básicos. En el caso del norte de Chaitén, hay organizaciones territoriales, como las juntas de vecinos "Chaitén Vive" y "Pillán". En cambio, en el sur las organizaciones que se destacan son funcionales -ej. se organizan para conseguir agua potableporque los residentes aún no han asegurado sus necesidades básicas. Según algunos vecinos, el nivel de participación social (en términos de Wilson, 2012) se ha reducido a medida que las organizaciones del sector norte han obtenido soluciones a sus demandas.

Lo mismo sucede con respecto a la percepción del riesgo. Tanto autoridades, líderes locales y otros chaiteninos comienzan a quitarle importancia a la presencia del volcán, cuya actividad ha decaído en el tiempo y por lo tanto se presta menos atención a las medidas de reducción de riesgo de desastres -tal como es explicado en otros casos por Wisner et al. (2004)- Por ejemplo, hacia finales del 2013, Chaitén no contaba con un Plan de Emergencia Comunal actualizado, esencial para establecer los roles y acciones tanto de las autoridades y la población en caso de emergencia. El último plan fue redactado el año 2010 (Municipalidad de Chaitén ONEMI, 2010). Según una autoridad comunal:

El Comité [Comité de Protección Civil o CPC] debería reunirse periódicamente para analizar el estado de nuestra preparación como ciudad, y asegurar que tenemos todo lo que necesitamos para reaccionar [...] lamentablemente no nos hemos reunido, nadie tiene tiempo. (Autoridad municipal anónima, 2013, com. pers).

Estas situaciones han erosionado la confianza de la comunidad en las autoridades y el Estado. Varios líderes locales consideran que podrían haber salvado a la ciudad de las inundaciones del 2008 si los pobladores se hubieran quedado durante la erupción. Si llegara el caso, no evacuaríamos de nuevo, y las futuras inundaciones se podrían mitigar reforzando el cauce del río Blanco (Líder local anónimo, 2013, com. pers.). Aunque se tomaron algunas medidas de mitigación para proteger el borde norte del nuevo cauce del río de posibles inundaciones y de la erosión, a la fecha de este trabajo no habían estudios disponibles sobre el borde sur del río, argumentándose que no se permite ninguna inversión pública en la zona. Con todo, las medidas de mitigación pueden disminuir

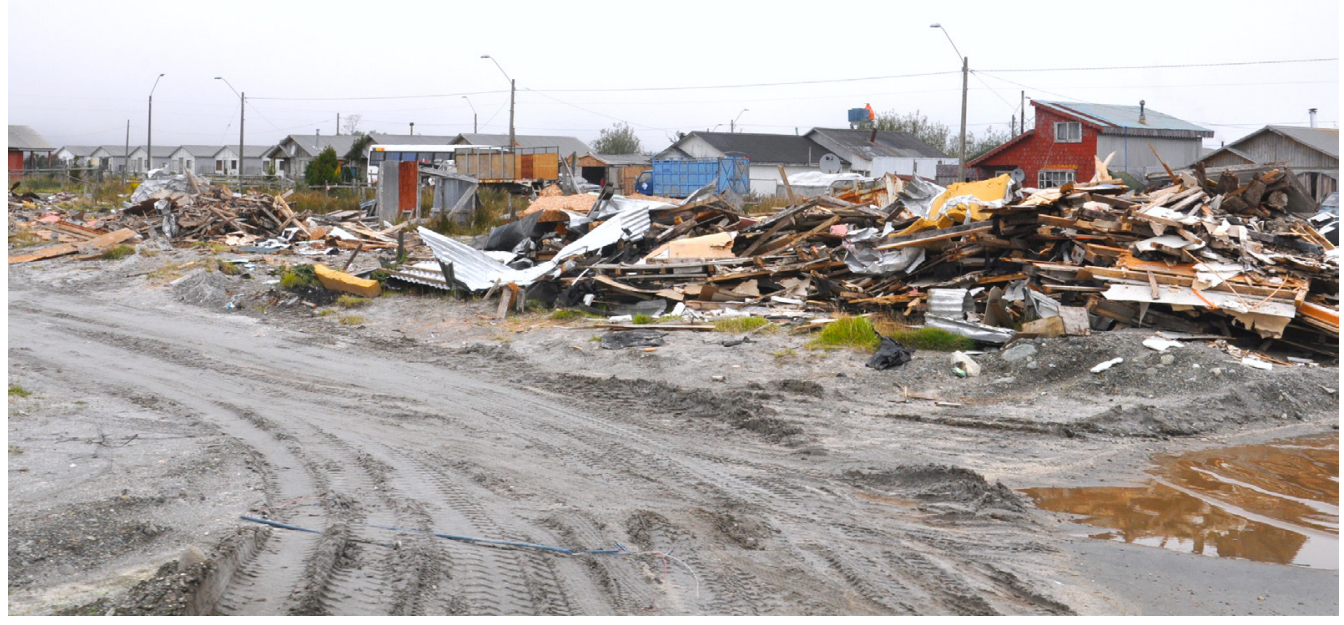

Fig. 4. Parte de las viviendas demolidas en el sector sur de Chaitén.

Fuente: Vicente Sandoval (2013). 
los daños de las inundaciones estacionales, sin embargo, los efectos de un evento volcánico intenso, así como inundaciones extremas, podrían ser catastróficas para toda la ciudad (INFRACON S.A., 2012). Desde esta perspectiva, los chaiteninos parecen estar más vulnerables a los peligros que antes de 2008, y no queda claro hasta qué punto ésta vulnerabilidad podría reducirse con medidas de mitigación.

Hacia finales de 2013, 4.000 chaiteninos vivían aún en exilio. De ellos, no todos regresarán finalmente a Chaitén, principalmente porque algunos de ellos han logrado integrarse eficazmente en otras ciudades y contextos. Por otro lado, la toma de decisiones y entredichos del gobierno continúa también hoy. Mientras la demolición de casas en el sector sur impide a más personas a establecerse allí, una reciente reunión entre el gobierno central y las autoridades locales dejó la puerta abierta para autorizar una ocupación formal de esta zona en el futuro (Rojas, 2013; Senado de la República de Chile, 2013). Si bien este anuncio parece positivo a la luz de las demandas de la población local, al momento de este estudio, no hubieron medidas concretas que hicieran pensar que el riesgo vaya a ser minimizado en la zona, por el contrario, el anuncio produjo la sensación de que antes o temprano el sector sur de Chaitén será declarado habitable, pudiendo alentar a nuevas ocupaciones, las cuales sin medidas concretas o planes para el desarrollo seguro del lugar, sólo provocarán el aumento de la población expuesta y con ello la vulnerabilidad y el riesgo.

\section{MÁS ALLÁ DEL VOLCÁN. LOS EFECTOS IMPREVISTOS SOBRE LA CAPACIDAD DE RECUPERACIÓN DE LA POBLACIÓN Y LA JUSTICIA AMBIENTAL}

Tal y como hemos revisado en Chaitén, las decisiones políticas adoptadas y sus efectos han tendido a erosionar la capacidad de las personas para aprender de y adaptarse a los impactos de los desastres. En este sentido, un ejemplo podría ser los efectos imprevistos de la entrega de la ayuda social llamada Bono Emergencia Chaitén y el subsidio a la vivienda entregado por el MINVU, y cómo estas ayudas promovieron el desarrollo de otro tipo de "dependencia del Estado". Durante los dos años que duraron éstas y otras ayudas, de acuerdo con algunos funcionarios locales, las personas se volvieron literalmente locas debido a la cantidad de dinero que repentinamente tuvieron que administrar. Antes del 2008, la gente solía vivir con mucho menos [...] Cuando recibieron 'esa' cantidad de plata [dinero], mucha gente la ocupó para vacaciones, viajes y ropa cara (Empleado público anónimo, 2013, com. pers.). Así, cuando los beneficios se agotaron, la mayoría de las personas se encontraron sin ahorros, con deudas y algunos con problemas psicológicos derivados de las deudas y el abandono (Paz, 2011; Sáez, 2009). En otras palabras, mientras que por un lado los beneficios pretendían ayudar y estaban pensados para reducir las dificultades de la población, por otro lado, éstos mismos beneficios pueden haber erosionado la resiliencia de la comunidad al facilitar una transformación drástica de sus medios de subsistencia y produciendo una dependencia del apoyo del Estado. Cambiar drásticamente los medios de subsistencia hacia una dependencia del estado podría poner en peligro la capacidad de la comunidad de Chaitén para reaccionar y recuperarse de los impactos negativos de futuros desastres (Schilderman \& Lyons, 2010).

La misma lógica aplica a otras circunstancias en que las acciones -o la falta de las mismas- de la comunidad y las autoridades acentúan una distribución desigual de los riesgos ambientales, vulnerabilidad y resiliencia. De este modo, Chaitén está dividida físicamente como consecuencia del nuevo curso del río Blanco, pero también, y más importante aún, dividida socialmente por el efecto imprevisto de las políticas y decisiones adoptadas que facilitaron el reasentamiento de la población en el sector sur. Por ejemplo, la incapacidad limitación institucional de la municipalidad y el gobierno regional para decidir la gestión de riesgos y promoción de la resiliencia revelan a la centralización institucional y política del país (Ostrom, 2010) como una barrera al desarrollo de respuestas locales y pertinentes en caso de desastre.

Una año después de la erupción del volcán Chaitén, el gobierno central (Presidencia de la República de Chile, 2009) creó y mantuvo una autoridad especial llamada "Delegada Presidencial", cuyo objetivo era apoyar a las familias 
desplazadas y facilitar el proceso de recuperación de la población de Chaitén. La creación de esta autoridad desencadenó que el flujo de decisiones $e$ información hiciera un bypass entre las autoridades regionales y locales, creando además tensiones sobre el destino de la ciudad de Chaitén y afectando finalmente la confianza de la población sobre su futuro (Allard, 2010). La tensión creada por esta nueva autoridad afectó la confianza de los ciudadanos en las autoridades; la errática decisión de declarar Chaitén muerto y más tarde habitable nuevamente, es un ejemplo de esto. La misma falta de certezas alimentó la esperanza de la población por ver al sector sur declarado habitable algún día, animándoles a 'ocupar' las casas del sector sur que, ahora en propiedad del Estado, ya no les pertenecen, perpetuando la incertidumbre entre esta población y el impacto que ésta tiene sobre la vulnerabilidad y el riesgo.

La demanda de la comunidad por un puente que conecte el norte y el sur, así como una protección para la ribera sur del río Blanco que detenga las inundaciones de invierno, siguen sin resolverse. Esto ocurre en la medida en que el 'riesgo volcánico' ofrece un poderosa excusa para que las autoridades (Schilderman \& Lyons, 2010) desatiendan el problema detrás de no llevar a cabo éstas medidas; las inundaciones de invierno en el sector sur empobrecen cada vez más a su población, haciéndolas más vulnerables, mientras que la falta de conectividad entre el norte y el sur limita el acceso a los servicios, creando graves desigualdades en la movilidad urbana, afectando de igual forma la vulnerabilidad de la población.

Contradiciendo la política de desalojamiento del sector sur, la Municipalidad de Chaitén, el Gobierno Regional (GORE) de Los Lagos y, recientemente, el gobierno central, han dado muestras en el cambio de postura sobre la legalidad del sector sur (Jara \& Baeza, 2012; Rojas, 2013; Senado de la República de Chile, 2013); un proyecto de alumbrado público para el año 2013 $y$ un proyecto de parque infantil aprobado $y$ ejecutado, son prueba de este potencial cambio de postura.

Mientras que la (in)justicia ambiental en Chaitén se puede expresar a través de la forma en que el sector sur está desproporcionadamente más expuesto a amenazas naturales que el sector norte, la capacidad de la población de Chaitén (en su conjunto) para recuperarse y adaptarse a nuevas amenazas ha sido y está siendo erosionada por decisiones políticas. Como se mencionó anteriormente, el Gobierno no ha comprendido correctamente las implicaciones de promover una gestión de riesgos aislada de la comunidad que habita el sector sur de Chaitén, la que afecta la resiliencia de la población total. En el sentido inverso, como Schilderman y Lyon señalan (2010), la (in)justicia ambiental expresada en la distribución espacial del riesgo también puede ser moldeada por las decisiones adoptadas para aumentar la resiliencia de la población. Por ejemplo, mientras que el gobierno regional inició las obras de demolición de viviendas en el sector sur con el fin de evitar más ocupaciones en la zona y reducir así el número de personas expuestas a peligros, todos los proyectos de nuevas vivienda en el norte se terminaron abruptamente (Baeza, 2013).

Procesos políticos como la toma de decisiones y cambios de autoridades pueden dar forma a la manera en que los procesos de justicia ambiental y resiliencia interactúan en un contexto post-desastre, como es en el caso de Chaitén. Tal como se presenta en este trabajo, los procesos y fuerzas política no deben ser considerados como meros factores subyacentes en la producción del riesgo y los desastres (Pelling, 2006; Wisner et al. 2004), sino por el contrario, activos progenitores del contexto que determinará los niveles de exposición, vulnerabilidad y la capacidad de la población para recuperarse y adaptarse a los impactos negativos de futuros desastres.

Finalmente, los efectos de las políticas y decisiones adoptadas sobre la comunidad en su conjunto en un contexto post-desastre se han traducido en la resistencia a futuras acciones y en una erosión de la confianza en las autoridades y el sector público. Asimismo, este trabajo sugiere que la pérdida de confianza de la comunidad, la desigual exposición de chaiteninos a los peligros, y el desigual acceso a los servicios públicos e infraestructura urbana, han afectado negativamente su capacidad para reaccionar y recuperarse futuros desastres. En este sentido, se destaca también que mejores procesos de negociación, planificación y coordinación entre la población y autoridades para construir consensos podrían influir positivamente 
en la resiliencia y la reducción de la vulnerabilidad de Chaitén.

\section{BIBLIOGRAFÍA}

Allard, P. (2010). Fables of Reconstruction: Chaitén, After the Volcano. Revista Harvard Review of Latina America, 9(2), 36-38.

Baeza, A. (2013). A cinco años de la erupción del volcán Chaitén: los logros y los proyectos de una ciudad que estuvo cerca de desaparecer. La Nación, 23 Abr, http://goo.gl/ocp47P, revisado el 22 Ago 2015.

Cifuentes, C. (2009). Delegada presidencial inicia proceso informativo por reubicación de Chaitén. La Tercera, 25 Feb, http://goo.gl/Vjloee, revisado el 19 Ago 2015.

CIMM TS Consultores (2010). Estudio de contaminación de cenizas y suelos en la ciudad de Chaitén y propuesta de medidas de mitigación y restauración. Santiago de Chile: SEREMI Salud Región de Los Lagos.

Cutter, S. L., Barnes, L., Berry, M., Burton, C., Evans, E., Tate, E., \& Webb, J. (2008). A place-based model for understanding community resilience to natural disasters. Global Environmental Change, 18(4), 598-606.

De la Barrera, F., Reyes-Paecke, S., \& Meza, L. (2011) Landscape analysis for rapid ecological assessment of relocation alternatives for a devastated city. Revista Chilena de Historia Natural, 84(2), 181-194.

El Mercurio (2013). Chaitén, a cinco años de dura erupción. El Mercurio, $29 \mathrm{Abr}$, http://goo.gl/MGsXmU, revisado el 21 Ago 2015.

El Mercurio (2010). Piñera corrige al intendente de Los Lagos y asegura que Nueva Chaitén se emplazará en Santa Bárbara. EMOL, 27 May, http://goo.gl/13E4bX, revisado el 21 Ago 2015.

EM-DAT (2015). Chile Country Profile. Brussels: Centre for Research on the Epidemiology of Disasters - CRED. EM-DAT 2015, 30 Ago, http://www.emdat.be/resultcountry-profile, revisado el 30 Ago 2015.

Galilea, S. (2009). Recursos "Emergencia Volcán Chaitén". Puerto Montt: GORE Los Lagos.

Gobierno Regional de Los Lagos - GORE (2008). Decreto Supremo $N^{\circ} 588$ del 02 de Mayo de 2008 que Señala como afectada por la catástrofe a la Provincia de Palena. Puerto Montt: Ministerio del Interior.

Gobierno Regional de Los Lagos - GORE (2009). Criterios en el Proceso de Asignación del Bono de desplazamiento víctimas del Volcán Chaitén. Puerto Montt: Ministerio del Interior.

Gobierno Regional de Los Lagos - GORE (2010). Criterios en el Proceso de Asignación del Bono de desplazamiento víctimas del Volcan Chiatén 2010. Puerto Montt: Ministerio del Interior.

González-Ferrán, O. (1995) Volcanes de Chile. Santiago de Chile: Instituto Geográfico Militar.

INFRACON S.A. (2012). Estudio de riesgo de sismos, volcanismo, remoción en masa, inundación por desborde de cauces y canales y maremotos para ocho localidades de la comuna de Chaitén, Informe Final. Santiago de Chile: SUBDERE.

Instituto Nacional de Estadísticas de Chile INE (2002). Censo. Santiago de Chile: INE.

Intendencia Región de Los Lagos (2010). Informó el intendente Montes: Declaración sobre habitabilidad de Chaitén. Gobierno de Chile, 10 Dic, http://goo.gl/gaasDr, revisado el 21 Ago 2015.

Jara, A., \& Baeza, M. (2012). Alcalde defiende gestión de atender a familias que viven en Chaitén Sur pese a no estar habilitado. BioBioChile.cl, 2 May, http://rbb. cl/2p16, revisado el 03 Ago 2015.

La Nación (2009). Edmundo Pérez Yoma: "Chaitén desgraciadamente ha muerto". La Nación, 20 Feb, http://goo.gl/61tqV3, revisado el 1 Ago 2015.

Lara, L. E. (2009). The 2008 eruption of the Chaitén Volcano, Chile: A preliminary report. Andean geology, 36(1), 125-129.

Mardones, R., Rueda, S., \& Guzmán, M. (2011). Tejiendo vínculos: una mirada a la organización "Renacer de Chaitén" de la tercera edad en un contexto de posdesastre. Cuadernos de Crisis y Emergencias 2(10), 19-40

Ministerio de Vivienda y Urbanismo MINVU (2010). Plan Maestro Ciudad de Chaitén: Informe Final. Santiago de Chile: MINVU.

Moreno, H., \& Lara, L. (2008). Peligros volcánicos potenciales del volcán Chaitén. Región de Los Lagos. Santiago de Chile: SERNAGEOMIN.

Moreno, H., Lara, L., Arenas, M., \& Derch, P. (2008). Evaluación preliminar de los peligros geológicos en la ciudad de Chaitén, Provincia de Palena, región de Los Lagos. Santiago de Chile: SERNAGEOMIN.

Municipalidad de Chaitén (2011). Reconstrucción de Chaitén. Santiago de Chile: Gobierno Regional de Los Lagos.

Municipalidad de Chaitén y ONEMI (2010). Plan de Emergencia Comuna de Chaitén. Chaitén: Ministerio del Interior.

Naranjo, J. A., \& Stern, C. R. (2004) Holocene tephrochronology of the southernmost part $\left(42^{\circ} 30^{\prime}-45^{\circ} \mathrm{S}\right)$ of the Andean Southern Volcanic Zone. Revista geológica de Chile 1(31), 224-240. 
Ostrom, E. (2010). Polycentric systems for coping with collective action and global environmental change. Global Environmental Change 20(4), 550-557.

Paz, V. (2011). Deudas, miedo y abandono, la otra cara del Chaitén. Revista Milcaos, 11 Ene, http://goo.gl/ GTN1LY, revisado el 11 Mar 2015.

Pelling, M. (2006). 'Natural disasters' as catalysts of political action. ISP/NSC briefing paper, 6(01), 4-6.

Pontificia Universidad Católica de Chile PUC, Observatorio de Ciudades PUC, Universidad Austral de Chile \& ARUP (2009). Consultoría para el desarrollo de lineamientos estratégicos de reconstrucción / relocalización y Plan Maestro conceptual post-desastre Chaitén. Santiago de Chile: PUC.

Presidencia de la República de Chile (2009). Cuenta Pública de la Delegada Presidencial en Chaitén. Santiago de Chile: Gobierno de Chile.

Quarantelli, E. L. (2004). Emergent behaviors and groups in the crisis time periods of disasters, Preliminary Paper, University of Delaware, Disaster Research Center working paper, 206, 1-8.

Ramirez, N. (2010). Alcalde de Chaitén: ¡En Talcahuano sabrán que nosotros llevamos dos años sin agua! EMOL, 10 May, http://goo.gl/EJzOLB, resviado el 11 Mar 2015.

Rojas, J. (2013). La reconquista de Chaitén. The Clinic, 23 Jul, http://goo.gl/4n7ttE, revisado el 30 Jul 2015.

Sáez, M. E. (2009). Chaitén: ¿Por qué resistimos? Un testimonio de las familias que permanecen en Chaitén, Parte 1 de 11, 2 May, http://youtu.be/X70jiVHsiMQ, revisado el
23 Jul 2015.

Senado de la República de Chile (2013). Recuperación de Chaitén. Senado de Chile, 1 Ago, http://www.senado. $\mathrm{cl} /$ recuperacion-de-chaiten-fisco-ha-adquirido-889inmuebles-e-invirtio-cerca-de-191-millones-de-dolares/ prontus_senado/2013-08-01/162329.html, revisado el 28 Abr 2015.

Schilderman, T., \& Lyons, M. (2010). Resilient dwellings or resilient people? Towards people-centred reconstruction. Environmental Hazards, 10(3-4), 218-231.

SERNAGEOMIN (2009). Erupción del Volcán Chaitén: Informes técnicos Mayo 2008-Marzo de 2009. Santiago de Chile: SERNAGEOMIN.

Silva, S. (2010). Piñera asegura que Chaitén será reubicado en localidad de Santa Bárbara. Radio Universidad de Chile, 28 May, http://goo.gl/zBOfac, revisado el 20 Jul 2015.

Sistema Nacional de Información Comunal SINIM. (2014). Informes y Bases de Datos. SINIM, http://www.sinim. gov.cl [revisado el 10 Mar 2014].

Walker, G. P., \& Bulkeley, H. (2006). Geographies of Environmental Justice. Geoforum 37(5), 655-659.

Wilson, G. A. (2012). Community resilience, policy corridors and the policy challenge. Land Use Policy, 31(0), 298310 .

Wisner, B., Blaikie, P., Cannon, T., \& Davis, I. (2004). At risk: natural hazards, people's vulnerability, and disasters. London; New York: Routledge. 
